\title{
Cross clinical-experimental-computational qualification of in silico drug trials on human cardiac Purkinje cells for proarrhythmia risk prediction
}

\author{
Cristian Trovato ${ }^{1}$, Marcel Mohr ${ }^{2}$, Friedemann Schmidt ${ }^{2}$, Elisa Passini ${ }^{1}$, and Blanca \\ Rodriguez ${ }^{3}$ \\ ${ }^{1}$ University of Oxford \\ ${ }^{2}$ Sanofi-Aventis Deutschland GmbH \\ ${ }^{3}$ Oxford University
}

February 16, 2022

\begin{abstract}
Background and Purpose Preclinical identification and understanding of drug-induced cardiotoxicity is still a major challenge. The ICH S7B Q\&A promote human in silico drug trials for proarrhythmia risk assessment. However, additional evidence is needed to support further regulatory impact and for their integration in the current preclinical assessment pipelines. This study aims to provide a comparative evaluation of drug-induced electrophysiological effects on in silico and in vitro cardiac Purkinje and to assess the accuracy of these models for clinical risk predictions. Experimental Approach The effects of 14 reference compounds were quantified in a population of in silico human cardiac Purkinje models, and compared with results obtained in in vitro rabbit Purkinje preparations. For each drug dose, five electrophysiological biomarkers were quantified at three pacing frequencies, and results compared with clinical proarrhythmia reports. Key Results i) In silico, repolarisation abnormalities in human Purkinje simulations predicted drug-induced arrhythmia for all risky compounds, showing higher predicted accuracy than rabbit experiments; ii) Drug-induced electrophysiological changes observed in human-based simulations showed a high degree of consistency with in vitro rabbit recordings at all pacing frequencies, and depolarisation velocity and action potential duration were the most consistent biomarkers; iii) discrepancies observed for dofetilide, sotalol and terfenadine are mainly caused by species differences between humans and rabbit. . Conclusion and Implications In this study we showed the high degree of consistency and higher accuracy of in silico methods compared to in vitro animal models, demonstrating the high regulatory impact of in silico trials for proarrhythmia prediction.
\end{abstract}

Cross clinical-experimental-computational qualification of in silico drug trials on human cardiac Purkinje cells for proarrhythmia risk prediction

Cristian Trovato $^{1 *}$, Marcel Mohr ${ }^{2}$, Friedemann Schmidt ${ }^{2}$, Elisa Passini $^{1}$, Blanca Rodriguez ${ }^{1}$

${ }^{1}$ Department of Computer Science, University of Oxford, Oxford, UK

${ }^{2}$ Sanofi-Aventis Deutschland GmbH, R\&BD Preclinical Safety, Frankfurt/Main, Germany

\section{* Corresponding Authors:}

Cristian Trovato Department of Computer Science, University of Oxford Wolfson Building, Parks Road OX1 3QD Oxford (UK) cristian.trovato@gmail.com

Blanca Rodriguez Department of Computer Science, University of Oxford Wolfson Building, Parks Road OX1 3QD Oxford (UK) blanca.rodriguez@cs.ox.ac.uk 
Word count: 3501

Tables: 2

Figures: 7

Manuscript Format : British English

\section{DATA AVAilABILITY STATEMENT}

The data that support the findings of this study are available from the corresponding author upon reasonable request. Some data may not be made available because of privacy or ethical restrictions.

\section{Conflicts of Interest Statement}

C.T., E.P., and B.R. declare no conflicts of interest. F.S., and M.M. are employees of Sanofi and may hold shares and/or stock options in the company.

\section{Author Contributions}

All the authors conceived and designed the study; C.T. designed the population of models for in silico drug assays, performed the simulations, analysed the data, prepared the figures, and drafted the manuscript; M.M. and F.S. provided the data for the reference compounds; C.T., E.P., M.M, F.S. and B.R. interpreted the results; all the authors edited and revised the manuscript and approved the final version.

\section{Acknowledgments: FUNDING STATEMENT}

C.T., E.P. and B.R. were respectively supported by the TransQST project (Innovative Medicines Initiative 2 Joint Undertaking under grant agreement No 116030, receiving support from the European Union's Horizon 2020 research and innovation programme and EFPIA), an NC3Rs Infrastructure for Impact Award (NC/P001076/1), and a Wellcome Trust Senior Research Fellowship in Basic Biomedical Sciences $(214290 / \mathrm{Z} / 18 / \mathrm{Z})$. These authors also acknowledge additional support from EPSRC Impact Acceleration Awards (EP/K503769/1), and the Oxford BHF Centre of Research Excellence (RE/08/004/23915, $\mathrm{RE} / 13 / 1 / 30181)$. The present study reflects only the author's view and the TransQST project is not responsible for any use that may be made of the information it contains.

We would like to thank Sanofi employees J.M. Chambard and V. Ballet for providing the experimental ion channel and Purkinje fibre assays. Many stimulating dialogues with Derek Leishman (Eli Lilly), and other contributors to the cardiac safety WP7 of the IMI TransQST were instrumental to this manuscript and are most gratefully acknowledged.

\section{What is already known}

Human in silico trials with ventricular models can predict clinical proarrhythmic risk with high accuracy

Rabbit cardiac Purkinje fibres are an in vitro preparation commonly used for preclinical cardiotoxicity screening

\section{What this study adds}

Human Purkinje simulations reached higher accuracy than in vitro rabbit experiments for clinical proarrhythmia prediction

Human-based simulations and rabbit-based experiments are highly consistent across different compounds, biomarkers, and pacing frequencies

\section{What is the clinical significance}

In silico trials can accelerate the design and development of safer and more efficient medicines 
Human-based modelling and simulation help the translation of preclinical proarrhythmia risk assessment to clinical scenarios $\backslash$ sout

\section{ABSTRACT \\ Background and Purpose}

Preclinical identification and understanding of drug-induced cardiotoxicity is still a major challenge. The ICH S7B Q\&A promote human in silico drug trials for proarrhythmia risk assessment. However, additional evidence is needed to support further regulatory impact and for their integration in the current preclinical assessment pipelines. This study aims to provide a comparative evaluation of drug-induced electrophysiological effects on in silico andin vitro cardiac Purkinje, and to assess the accuracy of these models for clinical risk predictions.

\section{Experimental Approach}

The effects of 14 reference compounds were quantified in a population ofin silico human cardiac Purkinje models and compared with results obtained in in vitro rabbit Purkinje preparations. For each drug dose, five electrophysiological biomarkers were quantified at three pacing frequencies, and results compared with clinical proarrhythmia reports.

\section{Key Results}

i) In silico, repolarisation abnormalities in human Purkinje simulations predicted drug-induced arrhythmia for all risky compounds, showing higher predicted accuracy than rabbit experiments; ii) Drug-induced electrophysiological changes observed in human-based simulations showed a high degree of consistency with in vitrorabbit recordings at all pacing frequencies, and depolarisation velocity and action potential duration were the most consistent biomarkers; iii) discrepancies observed for dofetilide, sotalol and terfenadine are mainly caused by species differences between humans and rabbit. .

\section{Conclusion and Implications}

In this study we showed the high degree of consistency and higher accuracy of in silico methods compared to in vitro animal models, demonstrating the high regulatory impact of in silicotrials for proarrhythmia prediction.

\section{KEYWORDS}

Human cardiac Purkinje, cardiac electrophysiology, drug-induced arrhythmias, in silico trials, computer modelling, drug safety testing, qualification.

\section{ABBREVIATIONS}

$\mathrm{AP}(\mathrm{s})$ Action potential(s)

APA Action potential amplitude

$\mathrm{APD}_{\mathrm{x}} \mathrm{AP}$ duration at $\mathrm{X} \%$ of repolarisation

BCL Basic cycle length

$\mathrm{dV} / \mathrm{dt}_{\mathrm{MAX}}$ Maximum upstroke velocity

EAD(s) Early after-depolarisation(s)

EOP Membrane potential at the end of repolarisation

$\mathrm{G}_{\mathrm{X}} \mathrm{I}_{\mathrm{X}}$ conductance

$\mathrm{IC}_{50}$ Drug concentration for $50 \%$ channel inhibition

$\mathrm{I}_{\mathrm{CaL}}$ L-type $\mathrm{Ca}^{2+}$ current 
$\mathrm{I}_{\mathrm{CaT}}$ T-type $\mathrm{Ca}^{2+}$ current

$\mathrm{I}_{\mathrm{f}}$ Funny current

$\mathrm{I}_{\mathrm{K} 1}$ Inward rectifier $\mathrm{K}^{+}$current

$\mathrm{I}_{\mathrm{Kr}}$ Rapid delayed rectifier $\mathrm{K}^{+}$current

$\mathrm{I}_{\mathrm{Ks}}$ Slow delayed rectifier $\mathrm{K}^{+}$current

$\mathrm{I}_{\mathrm{Na}}$ Fast $\mathrm{Na}^{+}$current

$\mathrm{I}_{\mathrm{NaK}} \mathrm{Na}^{+}-\mathrm{K}^{+}$pump current

$\mathrm{I}_{\mathrm{NaL}}$ Late $\mathrm{Na}^{+}$current

$\mathrm{I}_{\mathrm{NCX}} \mathrm{Na}^{+}-\mathrm{Ca}^{2+}$ exchanger current

$\mathrm{I}_{\text {to }}$ Transient outward $\mathrm{K}^{+}$current

$\mathrm{I}_{\text {sus }}$ Sustained outward $\mathrm{K}^{+}$current

PC(s) Purkinje cells

SS Steady State

TOP Take-off potential (membrane potential before depolarisation)

Trovato2020 Human cardiac Purkinje AP model published by Trovato et al. 2020

$\mathrm{V}_{\mathrm{m}}$ Membrane potential

\section{INTRODUCTION}

Preclinical assessment of drug-induced arrhythmia or proarrhythmia is a key requirement for pharmaceutical industries and regulators. This is particularly relevant for compounds showing a positive hERG (human Ether-à-go-go-Related Gene) signal, but also blocking other ionic channels (Gary Gintant, Sager, and Stockbridge 2016). The current ICH S7B/E14 guidelines have prevented new pro-arrhythmic drugs from entering the market, though, they have also led to premature termination of drug development (and potentially of valuable therapeutics) based solely upon either the hERG assay or through-QT study results (Lester and Olbertz 2016). hERG encodes the potassium channel related to the rapidly activating delayed rectifier potassium current $\left(\mathrm{I}_{\mathrm{Kr}}\right)$, which - when blocked - leads to prolongation of the QT segment of the ECG, and potentially to arrhythmia. Predicting proarrhythmia is challenging, due to the interplay of several ionic currents underlying the cellular electrical activity, i.e., the action potential (AP), and the complex drug-ionic channels interactions.

In vitro, in vivo, and ex-vivo animal models are widely used for preclinical proarrhythmia assessment, often considering metrics based on drug-induced AP prolongation as a surrogate of QT prolongation. Among these, cardiac Purkinje fibres obtained from dog or rabbit hearts, are one of the most established and ICH S7B-recommendedin vitro models for preclinical cardiotoxicity screening (EMEA 2006, Roche et al., 2010). However, species differences between animals and humans, limit the accuracy of animal models for clinical risk prediction, in addition to other limitations such as the hefty cost for the pharmaceutical industry and the ethical questions about the use of animal for research (Van Norman 2019).

In silico drug trials using human-based and biophysically-detailed models have proven to be a powerful technology for proarrhythmic risk predictions with high accuracy (Passini et al. 2017, 2019; Lancaster and Sobie 2016; Llopis-Lorente et al. 2020; Z. Li, Ridder, et al. 2019). Their use have been promoted by regulators such as the United States food and drug administration (FDA), that also launched the Comprehensive in vitro Proarrhythmia Assay (CiPA) initiative (Sager et al. 2014; Z. Li, Mirams, et al. 2019), and the European Medicines Agency (Musuamba et al. 2021), which established a task force on innovation for emerging therapies and technologies. 
Integration of human-based in silico trials in drug safety assessment requires a deep knowledge on their consistency with experimental and clinical recordings. We previously demonstrated how human in silico trials using ventricular cardiomyocytes reach higher prediction accuracy than animal models (Passini et al. 2017). However, a systematic and comprehensive evaluation of in silicodrug trials on cardiac Purkinje electrophysiology for proarrhythmia risk prediction is still missing. Therefore, the goal of this study is to compare drug-induced electrophysiological effects on a population of human-based in silico Purkinje models (Trovato et al., 2020) with preclinical in vitro experiments in commonly used rabbit Purkinje preparations, and to assess the accuracy of both models for predictions of clinical proarrhythmic risk, for a selection of 14 reference drugs. The population of models approach (Britton et al. 2013; Muszkiewicz et al. 2015; Varshneya, Mei, and Sobie 2021) scales the investigation from one single average model up to hundreds of models, to account for cell-to-cell electrophysiological variability and uncertainty. Therefore, with respect to the in vitro rabbit model, we hypothesised that human-based in silico drug trials improve predictions of drug-induced effects and clinical proarrhythmia risks, since they represent human pathophysiology and include a better representation of the variability in drug response.

\section{MATERIALS AND METHODS}

Figure 1 summarises the methodology used in the present study, later described in more details. Briefly, a diverse set of 14 reference compounds (Table 1) was investigated both in vitro and in silico to assess drug-induced changes in cardiac Purkinje electrophysiology and the drug safety profiles. First, automated patch clamp was used to quantify the half-maximal inhibitory concentrations (IC50s) for four cardiac ion channels, for each compound (Section 2.1.1). Then, experiments in rabbit Purkinje fibres (Section 2.1.2) and simulations using human Purkinje models (Section 2.2) were conducted to investigate drug-induced electrophysiological changes. Computational and experimental results were then compared to assess their consistency and ability to predict drug-induced AP changes. Different metrics (Section 2.3) were quantified from the in vitro and in silicoassays, and their ability to predict clinical proarrhythmia was also evaluated against the risk of Torsade de Pointes (TdP) arrhythmias reported on the CredibleMeds(r) repository (Woosley and Romer 1999), included in Table 1. 


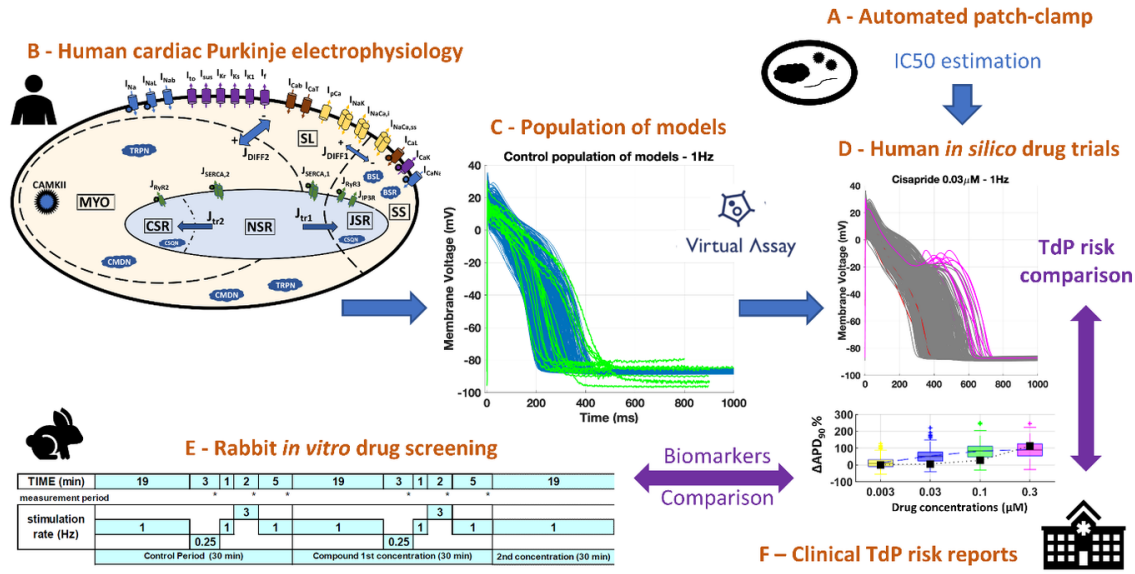

Figure 1. Combined experimental-computational pipeline used to perform this study. A: In vitro estimation of IC50s through automated patch clamp. B: Cartoon of the biophysically detailed computational model used to simulate human cardiac Purkinje electrophysiology (Trovato et al. 2020). C:Experimentally-calibrated population of 530 models generated using the Virtual Assay software (Oxford University Innovation (C) 2018); blue traces: computational models; green traces: experimental AP recordings from human healthy cardiac Purkinje cell (Nagy et al. 2015). D:Representative example of human in silico drug trials on the population of models, including AP traces (with drug-induced repolarisation abnormalities highlighted in pink) and biomarker boxplots. E: In silico results were compared against in vitro recordings from rabbit Purkinje fibres, obtained with the protocol depicted for multiple frequencies and concentrations.F: Both in vitro and in silico results were compared against the clinical TdP risk from the CredibleMeds@ repository 
((Woosley and Romer 1999)).

\subsection{Experimental data}

\subsubsection{In vitro ion channel data}

Four key human cardiac ion channels were selected: potassium channels hERG and hKv4.3 (modulating $\mathrm{I}_{\mathrm{Kr}}$ and the fast transient $\mathrm{K}^{+}$current, $\mathrm{I}_{\text {to }}$, respectively), L-type calcium channel hCav1.2 (modulating the L-type $\mathrm{Ca}^{2+}$ current, $\mathrm{I}_{\mathrm{CaL}}$ ), and sodium channel hNav1.5 (responsible for the fast $\mathrm{Na}^{+}$current, $\mathrm{I}_{\mathrm{Na}}$ ). These channels were previously identified as the minimum set of ion channels required for reliable in silico risk predictions (Zhou et al. 2020). Experimental in vitro patch clamp testing of ion channel inhibitions for the 14 reference compounds was internally performed on engineered immortalized cell lines. For in vitro hERG testing, HEK-293 cells were transfected stably with hERG cDNA and cultured in a 50:50 mix of Dulbecco's Modified Eagle's Medium and Ham's Nutrient Mixture F-12 (DMEM/F-12) supplemented with $10 \%$ foetal bovine serum (FBS). Each concentration was tested in a standard protocol for at least three cells (n [?] 3) at room temperature (21deg). Starting from $-80 \mathrm{mV}$, effects of sample compounds on the onset and steady state inhibition of hERG current were followed in response to a repeated pulse pattern, damping from steady $+20 \mathrm{mV}$ to $-80 \mathrm{mV}$. Obtained raw data was corrected for external effects at saturation concentration. Each compound was tested at eight appropriate concentrations in ascending order, and concentration limits were defined based on compound solubility, cytotoxicity or compound physicochemical properties. For the other ion channel targets (Cav1.2, Nav1.5 and Kv4.3) the screening was performed on the QPatch platform (Sophion, Ballerup, Denmark). HEK-293 cell lines expressing exogenous human targets were cultured according to internal protocols, in DMEM/F12 media supplemented with 10\% FBS. Standard 48 well plates were used in all experiments, and a standard voltage protocol mimicking elements of a ventricular action potential was applied at eight increasing concentrations to facilitate the determination of maximal inhibitory concentrations (IC50), with replicates. If no half-maximal inhibitory concentration was achieved in the specified concentration range, the result was interpreted as 'no inhibition'. Missing or inconclusive data in our studies were complemented from literature. All IC50 values used to perform in silico trials are reported in Table 1.

\subsubsection{In vitro drug assay on rabbit Purkinje fibres}

The effects of the 14 reference compounds on cardiac Purkinje electrophysiology were evaluated through microelectrode recordings from $(\mathrm{N}=6)$ isolated rabbit Purkinje fibres (male, New Zealand rabbits; 1.7 to 2.1 $\mathrm{kg} ; 7-10$ weeks of age).

The following biomarkers were quantified: take-off potential (TOP, in $\mathrm{mV}$ ), AP amplitude (APA, in $\mathrm{mV}$ ), maximal upstroke velocity $\left(\mathrm{dV} / \mathrm{dt}_{\mathrm{MAX}}\right.$, in $\left.\mathrm{V} / \mathrm{s}\right)$, AP duration at $50 \%$ and $90 \%$ of repolarisation $\left(\mathrm{APD}_{50}\right.$ and $\mathrm{APD}_{90}$ in $\left.\mathrm{ms}\right)$.

Test compounds were dissolved into dimethyl sulfoxide (DMSO) to obtain a stock solution. This solution was further diluted into 100\% DMSO to obtain solutions at different concentrations (as listed in Table 1) and added into the physiological solution.

The Purkinje fibres were first superfused with an oxygenated physiological solution containing (in mmol/L): $\mathrm{NaCl} 120 ; \mathrm{KCl} 4 ; \mathrm{MgCl} 2$ 1; NaH2PO4 1.8; NaHCO3 25; glucose 11; $\mathrm{CaCl} 2$ 1.8; $\mathrm{pH}=7.4$, at 36+-1degC. After a 30-minute control period, the test compound was evaluated at increasing concentrations that were sequentially applied, every 30 minutes. For the control period and each tested concentration, the fibres were stimulated at the basal rate of 1 pulse per second $(1 \mathrm{~Hz}$, normal pacing rate). In addition, stimulation rate was decreased from 1 pulse per second $(1 \mathrm{~Hz})$ to 1 pulse every 4 or 5 seconds $(0.25 \mathrm{~Hz}$ or $0.2 \mathrm{~Hz}$, low pacing rate) for 3 minutes, returned to 1 pulse per second for 1 minute and then increased to 3 pulses per second (3 $\mathrm{Hz}$, high pacing rate) for 2 additional minutes (between the 19th and the 25th minute) and finally decreased to 1 pulse per second $(1 \mathrm{~Hz})$ from the 25 th to 30 th minute as illustrated in Figure 1E.

The low stimulation rate favoured the occurrence of abnormal electrical events during the repolarization phase of the action potential, such as early after depolarisations (EADs). After testing the highest concentration, 
the physiological solution was superfused again to evaluate the reversibility of the drug effect (corresponding to a washout period).

Table 1. List of reference compounds, IC50 values recorded for four ionic currents, tested concentrations in rabbit preparations, and clinical proarrhythmic risk as reported by CredibleMeds (R) (Woosley and Romer 1999).

\begin{tabular}{llllll}
\hline Drug & IC50 $(\mu \mathrm{M})$ & IC50 $(\mu \mathrm{M})$ & IC50 $(\mu \mathrm{M})$ & IC50 $(\mu \mathrm{M})$ & Tested concentration $(\mu \mathrm{M})$ in rabbit preparations \\
\hline & $\mathbf{I}_{\mathbf{N a}}$ & $\mathbf{I}_{\mathbf{C a L}}$ & $\mathbf{I}_{\mathbf{t o}}$ & $\mathbf{I}_{\mathbf{K r}}$ & \\
Astemizole & 2.8 & 0.59 & 22 & 0.017 & $0.01,0.1,1,3$ \\
Bepridil & 3.1 & 6 & 13 & 0.19 & $0.1,0.3,1,3$ \\
Cisapride & - & 33 & - & 0.015 & $0.003,0.03,0.01,0.03$ \\
Clarithromycin & $163^{* *}$ & $103^{* *}$ & - & $62.5^{* *}$ & $1,2.4,10,30$ \\
Diltiazem & 15 & $0.76^{*}$ & 84 & 16.6 & $0.1,1,3,10,30$ \\
Disopyramide & - & 114 & - & $14.4^{*}$ & $0.3,1,3,10,30,100$ \\
Dofetilide & 94 & 204 & - & 0.047 & $0.0003,0.001,0.003,0.01$ \\
Nifedipine & 23 & 0.051 & 31 & 92 & $0.03,0.3,1,10$ \\
Quinidine & 35 & 2.9 & 15 & 1.26 & $0.1,1,3,10$ \\
Ranolazine & 101 & 156 & - & 24.5 & $0.3,3,10,30$ \\
Risperidone & 102 & 138 & 43 & 0.41 & $0.003,0.03,0.3$ \\
Sotalol & - & - & - & $86.4^{* *}$ & $0.3,1,3,10,30$ \\
Terfenadine & 3.3 & 2.2 & 68 & 0.17 & $0.03,0.32,1.44,5.34$ \\
Verapamil & 29 & $0.2^{*}$ & 58 & 0.6 & $0.1,1,3$ \\
\hline
\end{tabular}

IC50: drug concentration leading to $50 \%$ of current inhibition. $\mathbf{I}_{\mathbf{N a}}$ : fast $\mathrm{Na}^{+}$current; $\mathbf{I}_{\mathbf{C a L}}$ : L-type Ca ${ }^{2+}$ current; $\mathbf{I}_{\text {to }}$ : transient outward $\mathrm{K}^{+}$current; $\mathbf{I}_{\mathbf{K r}}$ : rapid delayed rectifier $\mathrm{K}^{+}$current. TdP risk : Torsade the Points risk: 1) Known risk; 2) Conditional risk; NC) Not classified, i.e., evidence was not enough to add it to any risk category. Dashes indicate no effect or IC50 much higher than tested concentrations (corresponding to a negligible block of the current). *: from (Kramer et al. 2013); **: from (Crumb et al. 2016); ${ }^{* * *}$ : from (Passini et al. 2019).

\subsection{Human in silico drug trials}

\subsubsection{Human cardiac Purkinje model}

Human cardiac Purkinje electrophysiology was simulated using the Trovato2020 model (Trovato et al. 2020), publicly available on the model repository of the Computational Cardiovascular Science Team (www.cs.ox.ac.uk/insilicocardiotox/model-repository). As shown in Figure 1B, the main ionic currents of this model are: fast and late $\mathrm{Na}^{+}$current $\left(\mathrm{I}_{\mathrm{Na}}\right.$ and $\mathrm{I}_{\mathrm{NaL}}$, respectively), $\mathrm{I}_{\mathrm{CaL}}$, T-type Ca ${ }^{2+}$ current $\left(\mathrm{I}_{\mathrm{CaT}}\right)$, $\mathrm{I}_{\text {to }}$, sustained outward $\mathrm{K}^{+}$current $\left(\mathrm{I}_{\text {sus }}\right)$, rapid and slow delayed $\mathrm{K}^{+}$rectifiers $\left(\mathrm{I}_{\mathrm{Kr}}\right.$ and $\mathrm{I}_{\mathrm{Ks}}$, respectively), inward $\mathrm{K}^{+}$rectifiers $\left(\mathrm{I}_{\mathrm{K} 1}\right)$, funny current $\left(\mathrm{I}_{\mathrm{f}}\right), \mathrm{Na}^{+}-\mathrm{Ca}^{2+}$ exchanger $\left(\mathrm{I}_{\mathrm{NCX}}\right)$ and $\mathrm{Na}^{+}-\mathrm{K}^{+}$pump $\left(\mathrm{I}_{\mathrm{NaK}}\right)$. The model was calibrated and evaluated against a wide set of AP recordings from human healthy Purkinje cells, and it is also able to reproduce the most common arrhythmia precursors at the cellular level, i.e., early and delayed afterdepolarisation (EADs and DADs, respectively).

\subsubsection{Population of human cardiac Purkinje models}

Starting from the Trovato2020 model, we developed a virtual population of human cardiac Purkinje cells, to incorporate biological variability. The population was designed similarly to what previously done in (Trovato et al. 2020), and using the well-established population of models methodology (Britton et al. 2013; Muszkiewicz et al. 2015). All simulations were performed using the Virtual Assay software (v.3.2 (C) 2018 Oxford University Innovation Ltd. Oxford, UK), a user-friendly software to perform in silico drug trials in population of models (Passini et al. 2020). An initial population of 1,000 models was constructed by sampling 
the 12 main ionic current conductances mentioned above $\left(\mathrm{I}_{\mathrm{Na}}, \mathrm{I}_{\mathrm{NaL}}, \mathrm{I}_{\mathrm{CaL}}, \mathrm{I}_{\mathrm{CaT}}, \mathrm{I}_{\mathrm{to}}, \mathrm{I}_{\mathrm{sus}}, \mathrm{I}_{\mathrm{Kr}}, \mathrm{I}_{\mathrm{Ks}}, \mathrm{I}_{\mathrm{f}}, \mathrm{I}_{\mathrm{K} 1}, \mathrm{I}_{\mathrm{NCX}}\right.$, $\left.\mathrm{I}_{\mathrm{NaK}}\right)$ in the range [50-200]\% of their baseline values, using Latin hypercube sampling (McKay, Beckman, and Conover 1979). First, models were paced individually for 1,000 beats to allow relaxation from the initial conditions and to reach the steady state at normal pacing $(1 \mathrm{~Hz})$. For each model, nine AP biomarkers were computed on the last simulated beat, including all the ones already described above $\left(\mathrm{APD}_{90}, \mathrm{APD}_{50}\right.$, $\mathrm{dV} / \mathrm{dt}_{\mathrm{Max}}$, TOP and APA), and also AP duration at $10 \%, 25 \%$, and $75 \%$ of repolarisation $\left(\mathrm{APD}_{10}, \mathrm{APD}_{25}\right.$, $\left.\mathrm{APD}_{75}\right)$ and the "end of potential" voltage (EOP). Only models exhibiting all AP biomarkers within the experimental ranges measured in healthy human Purkinje cells (Trovato et al. 2020; Nagy et al. 2015) and no repolarisation abnormalities (i.e., EADs or DADs) were retained in the final calibrated population, for a total of 530 models. All models in the final population were also paced for further 150 beats at slow pacing $(0.2$ or $0.25 \mathrm{~Hz})$ and fast pacing $(3 \mathrm{~Hz})$, to obtain control AP biomarkers for all the frequencies used in the in vitroexperiments.

\subsubsection{Human in silico drug trials}

Drug-induced inhibition of the different ion channels was simulated using a simple pore-block model, with the experimental IC50 and drug concentrations reported in Table 1 for $\mathrm{I}_{\mathrm{Na}}, \mathrm{I}_{\mathrm{CaL}}, \mathrm{I}_{\mathrm{Kr}}$ and $\mathrm{I}_{\mathrm{to}}$, and Hill coefficients equal to 1 . Figure 2 shows a visual representation of the residual currents following drug administration, for each compound and each concentration.

Starting from the steady state described above, the models were paced for further 150 beats at each frequency including the drug effects. Extracellular concentrations were set as in the in vitro rabbit experiments, and the same AP biomarkers were computed on the last simulated beat. Repolarisation abnormalities were detected as positive derivatives of the membrane voltage over time, occurring after 150 second, as in (Trovato et al. 2020). AP biomarkers were not computed for models showing abnormalities.

All simulations were performed on a regular Desktop Computer (Intel (R) Core (TM) i5-4670S CPU @ $3.10 \mathrm{GHz}$ RAM: $8 \mathrm{~GB}, 64$-bit Windows 10). The time required to simulate one drug at one concentration (150 beats) in a population of 530 models was 12,17 , and 35 minutes, for simulations at 3,1 , and $0.2 \mathrm{~Hz}$, respectively. 

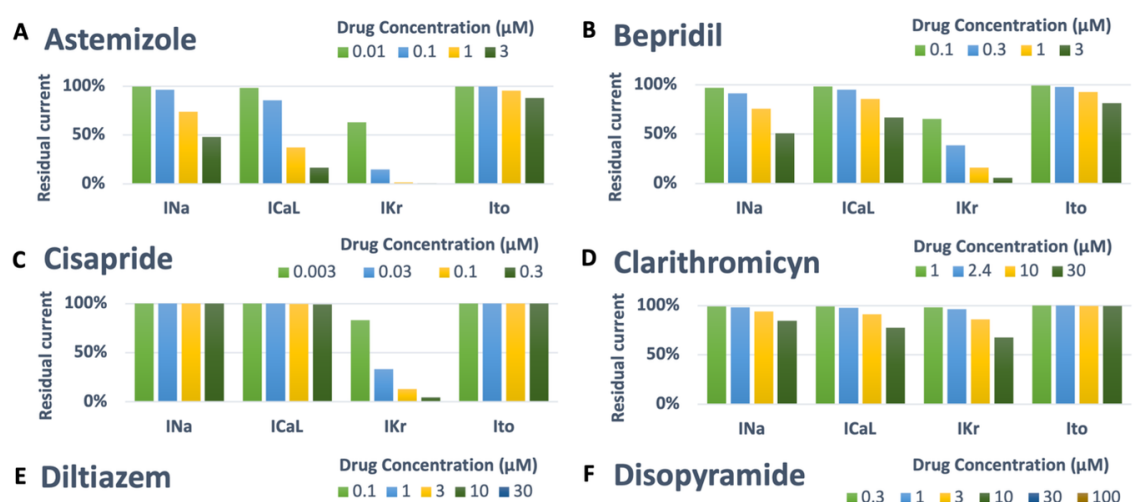

E Diltiazem $\quad \begin{aligned} & \text { Drug Concentration }(\mu M) \\ & m 0.1 \pm 1=3 \approx 10 \approx 30\end{aligned}$
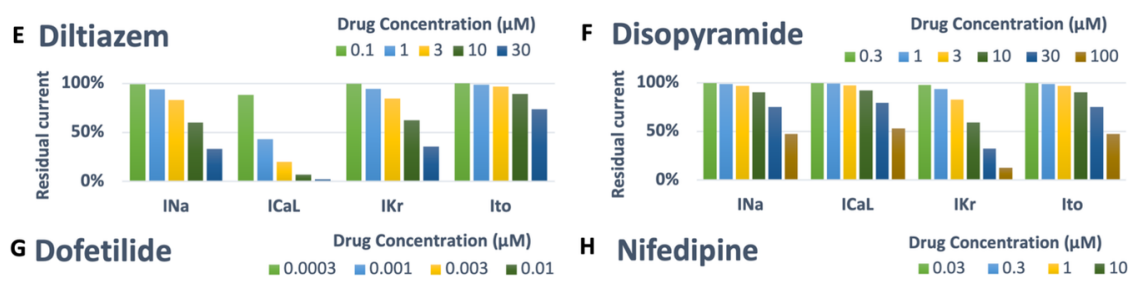

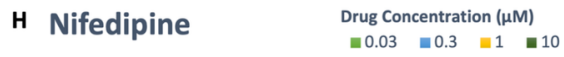

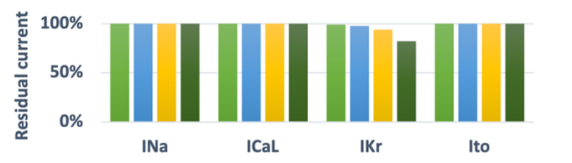

I Quinidine Drug Concentration $(\mu \mathrm{M})$
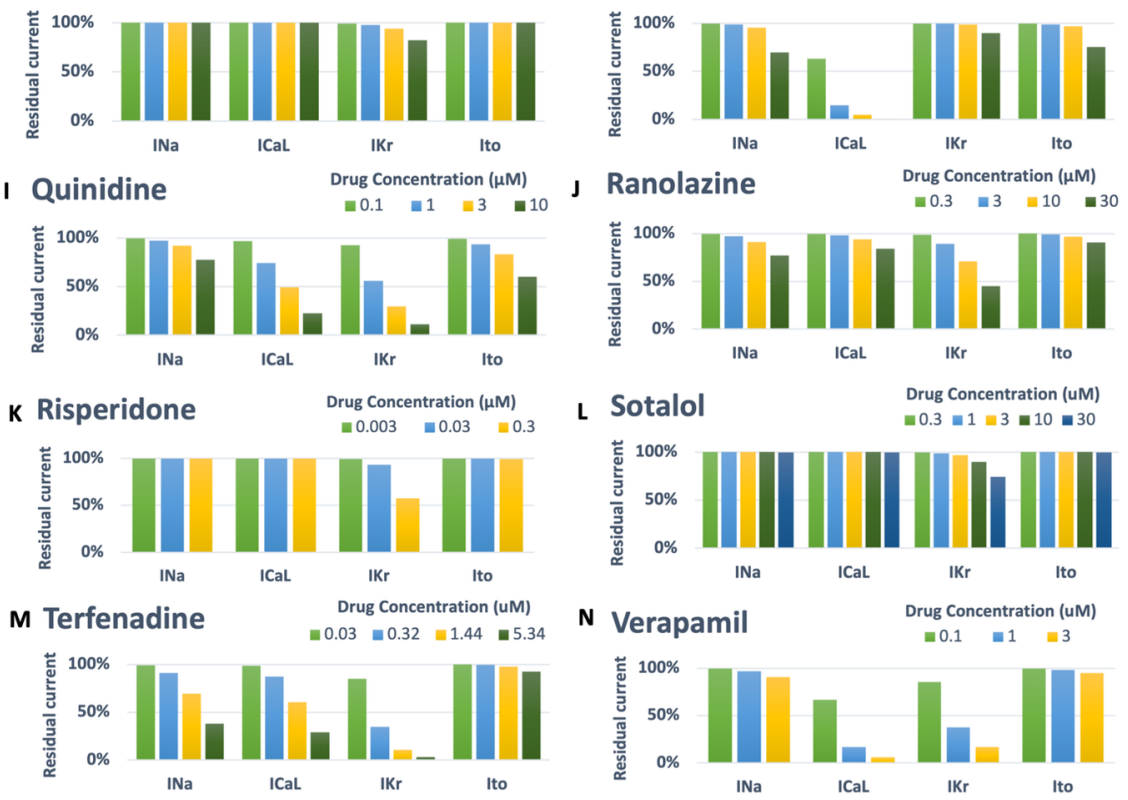

Figure 2. Summary of drug-induced effects on the cardiac ion channels, computed with a simple pore-block model. Each panel shows one of the 14 reference compounds, with the different bars representing the residual current after drug-application (in percentage), for each ion channel and drug concentration.

\subsection{Metrics for comparison of experiments, simulations, and clinical evidence}

We first compared the mean of simulated and experimental AP biomarkers in control conditions (no drug) for all pacing frequencies, to assess the consistency between in silico and in vitro models. Then, we compared the results for each compound at each concentration and pacing frequency against control. Results are shown as percent variations of the means, for both experiments and simulations.

For proarrhythmia risk assessment, we considered two different metrics. The first is the occurrences of 
drug-induced abnormalities, as previously used by (Passini et al. 2017). In summary, compounds inducing repolarisation abnormalities in at least one model of the in silico population or one cell of the in vitro rabbit assay, at any of the tested concentrations, were classified as risky, whereas they were classified as safe if all models/cells fully repolarised at slow pacing. The second is a metric based on APD $\mathrm{P}_{90}$ prolongation, since it is one of the most common biomarkers still used to discriminate between safe and proarrhythmic compounds in the current preclinical pipelines, even though it is not very specific (Champeroux et al. 2005; Redfern et al. 2003). In particular, we considered a mean $\mathrm{APD}_{90}$ prolongation higher than $10 \%$ as a warning for possible drug-induced proarrhythmic effects. Classification results based on these metrics for in vitro rabbit and in silicohuman trials were then compared against the clinical risk as reported by CredibleMeds@) (Woosley and Romer 1999), which divides drugs in multiple categories, based on TdP risk. As shown in Table 1, the 14 reference compounds belong either to category 1 (high risk: the drug prolongs the QT interval and is clearly associated with a known proarrhythmia risk, even when taken as recommended), 2 (conditional risk: the drug is associated with TdP but only under certain circumstances, e.g., overdose or interaction with other drugs), or NC (not classified - the drug was reviewed by CredibleMeds@ but the evidence available was not enough to assign it to any of the previous categories. For the purpose of this study, drugs in categories 1 and 2 were considered risky, while drugs in category $\mathrm{NC}$ were considered safe.

Finally, to evaluate the consistency between experiments and simulations, we defined a third metric, based on the mean of drug-induced percent variations in AP biomarkers for each tested drug and concentration: i) strong agreement, if the trend (increase/decrease) was the same, and the difference between the in vitro and in silico means was equal or less than $15 \%$; ii) qualitative agreement, if the trend (increase/decrease) was the same, but the difference between the in vitro and in silico means higher than $15 \%$; iii) disagreement, if the trend was different, regardless of the magnitude of the mean difference.

\section{RESULTS}

\subsection{Simulated and experimental AP biomarkers in control}

Table 2 reports mean and standard deviation of each AP biomarker in control conditions for the in vitro rabbit experiments $(\mathrm{n}=6)$ and the population of human in silico models $(\mathrm{n}=530)$ at slow, normal, and fast pacing. $\mathrm{APD}_{90}$ (and to a lesser extent $\mathrm{APD}_{50}$ ) is larger in rabbit experiments compared to human simulations at all pacing frequencies and, to a greater extent, at slow pacing. Decreasing the pacing frequency results in larger APD prolongation in rabbit than human Purkinje cells, in agreement with what has been previously measured in rabbit (W. Li et al. 2016) and human (Nagy et al. 2015) Purkinje cells. Both dV/dtmax and APA were also larger in rabbit experiments than human simulations: again, this is in agreement known interspecies differences (Cohen, Bean, and Tsien 1984; Nagy et al. 2015; Roche et al. 2010). No major differences were observed for TOP.

Table 2. Experimental and simulated AP biomarkers in control conditions (no drug) at slow pacing (0.2), normal pacing $(1 \mathrm{~Hz})$ and fast pacing $(3 \mathrm{~Hz})$.

Control

\begin{tabular}{lllllll}
\hline & Slow pacing & Slow pacing & Normal pacing & Normal pacing & Fast pacing & Fast pacing \\
& Exp & Sim & Exp & Sim & Exp & Sim \\
APD $_{90}$ & $432 \pm 119$ & $292 \pm 65$ & $310 \pm 52$ & $281 \pm 55$ & $216 \pm 24$ & $212 \pm 27$ \\
APD $_{50}$ & $311 \pm 107$ & $215 \pm 59$ & $239 \pm 53$ & $210 \pm 50$ & $162 \pm 24$ & $149 \pm 22$ \\
dV $/ d_{\text {Max }}$ & $627 \pm 80$ & $363 \pm 73$ & $616 \pm 72$ & $419 \pm 79$ & $595 \pm 62$ & $391 \pm 79$ \\
APA & $128 \pm 3$ & $107 \pm 4$ & $129 \pm 3$ & $112 \pm 4$ & $129 \pm 3$ & $113 \pm 4$ \\
TOP & $-89 \pm 1$ & $-84 \pm 1$ & $-91 \pm 1$ & $-87 \pm 1$ & $-92 \pm 1$ & $-88 \pm 1$ \\
\hline
\end{tabular}

Data shown as mean + standard deviation. Exp: experiments in in vitro rabbit cardiac Purkinje fibres $(\mathrm{n}=6)$; Sim: simulations in human cardiac Purkinje AP models $(\mathrm{n}=530)$. APD $\mathrm{X}$ : AP duration at $\mathrm{X} \%$ of repolarisation; $\mathrm{dV} / \mathrm{dt}_{\mathrm{MAX}}$ : maximal upstroke velocity; APA: AP amplitude; TOP: take-off potential. 


\subsection{Proarrhythmic risk assessment based on drug-induced repolarisation abnormalities}

The occurrence of drug-induced abnormalities in repolarisation was quantified in both experiments and simulations and is reported in Figure 3 ( $11^{\text {th }}$ and $12^{\text {th }}$ columns, respectively). These data were used to classify drugs as safe or risky, as described in the Methods section, and the results are shown in Figure 4A (left panel). Human in silico drug trials correctly classified all drugs (accuracy $=100 \%$ ), while the in vitro rabbit models achieved an accuracy of $79 \%$. Indeed, in rabbit preparations, EADs were observed for only 8 out of 11 risky compounds, while no EADs were observed for bepridil, ranolazine, and terfenadine, despite the wide range of concentrations explored. Diltiazem, nifedipine and verapamil did not induce any EADs in silico nor in vitro, and were correctly classified as safe.

Figure 5A reports a comparison between experimental and simulated AP traces for three illustrative compounds. Astemizole and cisapride (Figure 5A, left and central panels respectively) induced EADs in both simulations and experiments. For astemizole, EADs occurred at lower concentrations and largely disappeared at higher concentrations, due to the concurrent strong (>50\%) inhibition of $\mathrm{I}_{\mathrm{CaL}}$ (Figure 2, panel A), whereas for cisapride EADs were observed up to the highest tested concentration, with increasing duration. Diltiazem (Figure 5A, right panel) lowered the AP plateau and increased the AP duration in both experiments and simulations, but it did not induce any repolarisation abnormalities, in line with its safe profile.

Figure 5B shows experimental and simulated AP traces for the tree risky compounds that were correctly identified by the human simulations based on EADs occurrence (bottom panels), but misclassified by the rabbit experiments, i.e., bepridil, ranolazine and terfenadine. Simulations with bepridil showed EADs duration increasing with drug concentrations, similarly to cisapride, despite a mild $(<50 \%)$ inhibition of $\mathrm{I}_{\mathrm{CaL}}$ (Figure 2, panel B). Simulations with ranolazine showed EADs only at the highest concentration tested (Figure 5B, central panel), in line with its conditional proarrhythmic profile. Finally, simulations of terfenadine (Figure $5 \mathrm{~B}$, right panel) displayed EADs only at lower concentrations, similarly to astemizole.

From a mechanistic point of view, models developing EADs were characterised by low repolarisation reserve as previously investigated both in human cardiac Purkinje and ventricular models (Trovato et al. 2020; Passini et al. 2017) 


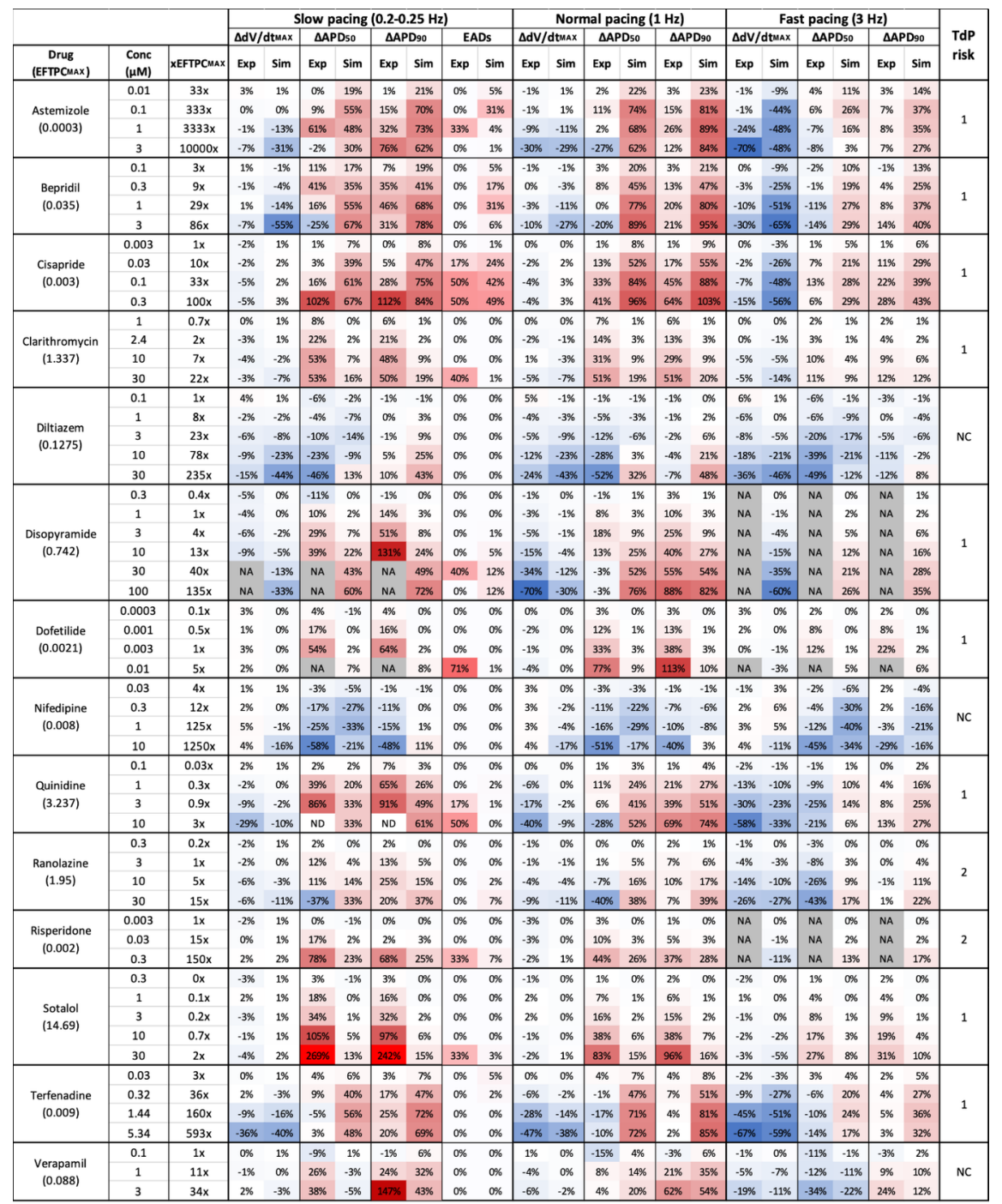

Figure 3. Comparison between clinical TdP risk and percent drug-induced AP changes in human simulations and rabbit experiments for each drug, at different concentrations and pacing frequencies.

Columns description from left to right: $1^{\text {st }}$ : drug name and maximum effective free plasma concentrations $\left(\mathrm{EFTPC}_{\mathrm{MAX}}\right.$, in $\left.\mu \mathrm{M}\right) ; 2^{\text {nd }}$ : tested drug concentrations; $3^{\text {rd }}$ : ratio between tested concentrations and EFTPC $_{\text {MAX }}$; from $4^{\text {th }}$ to 9 th: experimental and simulated AP biomarkers changes at slow pacing; $10^{\text {th }}$ to $11^{\text {th }}$ : percentage of EADs occurrences in simulations and experiments at slow pacing; from $12^{\text {th }}$ to $23^{\text {rd }}$ : experimental and simulated AP biomarkers changes at normal and fast pacing; $24^{\text {th }}$ : drug risk category based on CredibleMeds ( (Woosley and Romer 1999). NA: data not available. Changes are shown as percentage with respect to control conditions. Colours indicates biomarker increases (red) or decreases (blue) and are scaled from the minimum to the maximum percent variations for each biomarker at each pacing frequencies. 
A

Drug-induced proarrhythmia predictions based on:

\begin{tabular}{|c|c|}
\multicolumn{2}{c}{ EADs occurren } \\
\multicolumn{2}{|c|}{ Simulations } \\
\hline \begin{tabular}{c|c} 
True + \\
11
\end{tabular} & $\begin{array}{c}\text { True - } \\
11\end{array}$ \\
\hline False + & False - \\
0 & 0 \\
\hline
\end{tabular}

Accuracy: $100 \%$ Sensitivity: $100 \%$ Specificity: $100 \%$
Experiments

\begin{tabular}{|c|c|}
\hline True + & True - \\
$\mathbf{8}$ & $\mathbf{3}$ \\
\hline False + & False - \\
0 & 3 \\
\hline
\end{tabular}

Accuracy: $79 \%$ Sensitivity: $73 \%$ Specificity: $100 \%$
$A P D_{90}$ prolongation at $1 \mathrm{~Hz}$

Simulations

\begin{tabular}{|c|c|}
\hline $\begin{array}{c}\text { True + } \\
11\end{array}$ & $\begin{array}{c}\text { True - } \\
1\end{array}$ \\
\hline $\begin{array}{c}\text { False }+ \\
2\end{array}$ & $\begin{array}{c}\text { False - } \\
0\end{array}$ \\
\hline
\end{tabular}

Accuracy: $86 \%$ Sensitivity: $100 \%$ Specificity: $33 \%$
Experiments

\begin{tabular}{|c|c|}
\hline $\begin{array}{c}\text { True }+ \\
10\end{array}$ & True - \\
\hline False + & False - \\
\hline 1 & 1 \\
\hline
\end{tabular}

Accuracy: $86 \%$

Sensitivity: $91 \%$ Specificity: $67 \%$

\section{B Consistency between human in silico and rabbit In vitro $\mathrm{APD}_{90}$-based safety predictions}

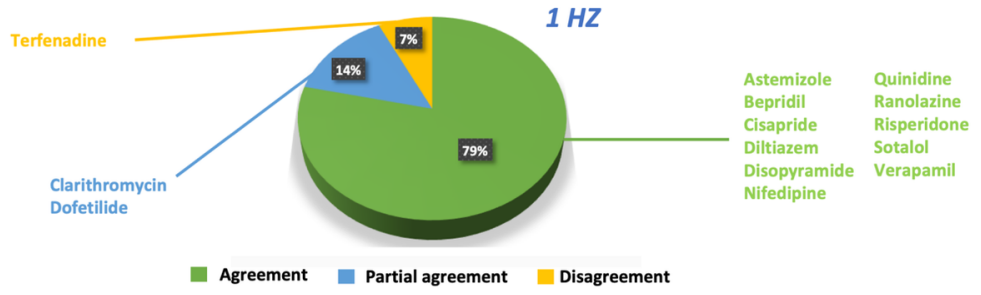

Figure 4. Proarrhythmic risk predictions using in silicohuman or in vitro rabbit models. A) Confusion matrix for in silico (green) and in vitro (blue) predictions, compared to clinical report of proarrhythmia, based on EADs occurrences (left panel) or $\mathrm{APD}_{90}$ (right panel); +: Risky drug; -: Safe drug. B) Consistency between in silico and in vitro drug safety predictions based on $\mathrm{APD}_{90}$ prolongation at $1 \mathrm{~Hz}$. The pie chart represents the percentage of compounds showing agreement for the majority of the concentration tested (green), agreement for at least half of the tested concentrations (blue), or disagreement (yellow). 

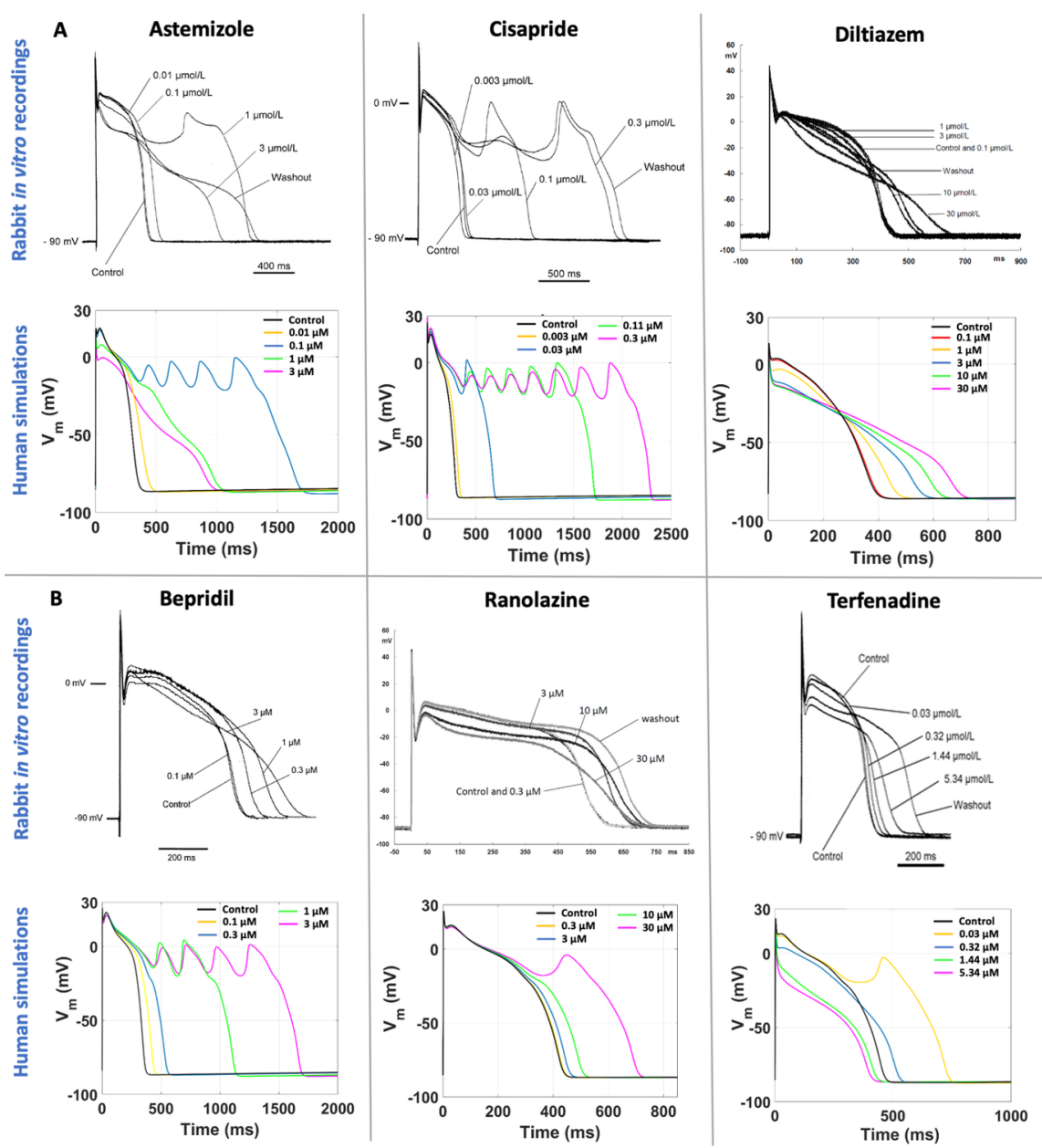

Figure 5. Comparison between human in silico andin vitro rabbit AP traces at slow pacing, for six illustrative compounds. In each section, experimental recordings are at the top, and simulated traces at the bottom. One representative cell/model is shown for each drug. A) Three explicative compounds showing EADs in both simulations and experiments: astemizole (left), cisapride (centre), diltiazem (right). B) The three compounds showing disagreement in EADs occurrence between in silico and in vitro results, i.e., EADs were observed only in simulations and not in experiments: bepridil (left), ranolazine (centre), terfenadine (right).

3.3 Proarrhythmia risk assessment through AP prolongation: consistency between experiments, simulations, and clinical reports 
Since $\mathrm{APD}_{90}$ is widely used in preclinical safety studies, we also evaluated drug-induced changes in $\mathrm{APD}_{90}$ in rabbit experiments and human simulations. Percentage changes compared to control conditions are reported in Figure 3, while Figure 4A (right panel) shows the confusion matrices obtained by considering as risky the drugs showing an average $\mathrm{APD}_{90}$ prolongation at $1 \mathrm{~Hz}$ greater than $10 \%$, as described in Section 2.3. Based on this metric, human in silico drug trials correctly classified all risky drugs, even though two safe drugs resulted false positives, yielding a total accuracy of $86 \%$. This is due to the fact that diltiazem and verapamil showed significant AP prolongation at high concentrations (78x and $11 \mathrm{x}$, respectively) and were therefore classified as risky. This AP prolongation is due to the large $(>50 \%)$ hERG blockade at concentrations far from the EFTPCmax. In vitro rabbit assays produced the same overall accuracy, even though it was achieved with one false positive (verapamil) and one false negative (terfenadine), the latter showing very little AP prolongation at all tested concentrations tested (up to 593x $\mathrm{EFTPC}_{\mathrm{MAX}}$ ), despite is high TdP risk.

The pie chart in Figure 4B summarises the consistency between in silico and in vitro predictions based on $\mathrm{APD}_{90}$ prolongation at $1 \mathrm{~Hz}$ : out of 14 reference compounds, 11 are in agreement, 2 are in partial agreement, and only 1 is in disagreement (categories defined as described in Section 2.3). Disagreement was observed for terfenadine, which induced up to $85 \%$ AP prolongation in simulations, and only up to $7 \%$ in in vitro rabbit experiments.

Partial agreement was observed for clarithromycin and dofetilide. As shown in Figure 3, both in silico and in vitro results show a dose-dependent AP prolongation for both drugs. However, the percentage changes observed in rabbit experiments are much larger than the ones observed in simulations (10\% vs $113 \%$ for dofetilide and $20 \%$ vs $51 \%$ for clarithromycin, respectively, at the maximum tested concentration). A similar behaviour was also observed for sotalol (Figure 3), and it can be related to known species differences between humans and rabbit in the response to hERG blockers. It is also worth to notice that in vitro experiments for dofetilide and sotalol were performed in a narrow range of concentrations (up to 5x and 2x the EFTPCmax, respectively), limited by the solubility of the drugs. This limitation can be easily overcome with in silico trials, and - when simulating higher concentrations - we actually observed larger AP prolongations in the population of models: $+57 \%$ for dofetilide $0.1 \mu \mathrm{M}$ and $+39 \%$ for sotalol at $100 \mu \mathrm{M}$.

\subsection{Detailed comparison of human in silico and rabbitin vitro drug trials}

Figure 3 shows experimental and simulated results for each drug, at each concentration and pacing frequency. Only $\mathrm{dV} / \mathrm{dt}_{\mathrm{MAX}}, \mathrm{APD}_{50}$ and $\mathrm{APD}_{90}$ are included, since minor drug-induced effects were observed for TOP and APA, in both experiments and simulations. Furthermore, $\mathrm{dV} / \mathrm{dt}_{\mathrm{MAX}}, \mathrm{APD}_{50}$ and $\mathrm{APD}_{90}$ are good markers of $\mathrm{I}_{\mathrm{Na}}, \mathrm{I}_{\mathrm{CaL}}$ and $\mathrm{I}_{\mathrm{Kr}}$ blocks, respectively, since they capture different phases of the AP dynamic: $\mathrm{dV} / \mathrm{dt}_{\mathrm{MAX}}$ is strongly dependent on $\mathrm{I}_{\mathrm{Na}}$, whereas $\mathrm{I}_{\mathrm{CaL}}$ modulates the AP plateau and $\mathrm{APD}_{50}$, and $\mathrm{I}_{\mathrm{Kr}}$ is mainly responsible for the later phase of repolarisation, captured by $\mathrm{APD}_{90}$.

Figure 6 summarises the comparison between experiments and simulations, based on the results from Figure 3 and using the metric defined in Section 2.3: each pie chart includes (for each biomarker, at each pacing frequency) the percentage of drug concentrations in either strong agreement (green), qualitative agreement (green), or disagreement (yellow), across all drug trials. These results clearly demonstrate a high degree of consistency between experiments and simulations, across all drugs, concentrations, pacing frequencies, and AP biomarkers.

Results for $\mathrm{dV} / \mathrm{dt}_{\mathrm{MAX}}$ show almost total agreement ( $98 \%$ for all pacing frequencies): the only drug showing disagreement is nifedipine at $10 \mu \mathrm{M}$, which slightly increase $\mathrm{dV} / \mathrm{dt}_{\mathrm{MAX}}(4 \%)$ in rabbit and reduces it in humans $\left(-16 \%,-17 \%\right.$ and $-11 \%$ at slow, normal and fast pacing, respectively). Drug-induced $\mathrm{APD}_{90}$ changes also shows a high degree of consistency (94-96\%) between experiments and simulations, even though - compared to $\mathrm{dV} / \mathrm{dt}_{\mathrm{MAX}}$ - a larger portion of drugs showed qualitative rather than strong agreement. Disagreement was only found in $6 \%, 5 \%$, and $4 \%$ of drug concentrations, for slow, normal, and fast pacing, respectively. Results for $\mathrm{APD}_{50}$ also show good agreement (71-83\%), despite the percentage of disagreement is higher compared to $\mathrm{dV} / \mathrm{dt}_{\mathrm{MAX}}$ and $\mathrm{APD}_{90}$. This difference could be explained by the differences in AP morphology between humans and rabbit: rabbit APs have a more pronounced spike compared to in silico models and this affects 
the voltage threshold to compute the $\mathrm{APD}_{50}$.

Disopyramide showed larger $\mathrm{APD}_{90}$ at slow pacing in rabbit experiments compared to simulations, and smaller $\mathrm{APD}_{50}$ at $1 \mathrm{~Hz}$ and high concentration (Figure $7 \mathrm{~A}$ ).

Results for quinidine and risperidone were strongly consistent at $1 \mathrm{~Hz}$, whereas - at slow pacing - a larger AP prolongation was observed in experiments compared to simulations. This could be due to differences in rate-dependent drug-induced effects on $\mathrm{I}_{\mathrm{CaL}}$ and $\mathrm{I}_{\mathrm{Kr}}$ between human and rabbit as reported in (Nagy et al. 2015; W. Li et al. 2016).

For all tested concentrations, astemizole, bepridil (Figure 7B) and cisapride induced a larger increase in $\mathrm{APD}_{90}$ in simulations compared to rabbit preparations, especially at $1 \mathrm{~Hz}$ and very high concentrations. For these three compounds, $\mathrm{dV} / \mathrm{dt}_{\mathrm{MAx}}$ predictions were strongly in agreement with the experiments at $1 \mathrm{~Hz}$, whereas, at both slow and fast pacing, they lead to a larger reduction in $\mathrm{dV} / \mathrm{dt}_{\mathrm{MAX}}$ in simulations than experiments. For astemizole and bepridil, $\mathrm{APD}_{50}$ was the less consistent biomarker, especially at high frequency, since simulations showed marked $\mathrm{APD}_{50}$ increased which was not observed in experiments. Simulations and experiments for clarithromycin showed agreement at fast pacing, whereas at slow pacing, simulations were just qualitatively in agreement, showing smaller AP prolongation compared to experiments. Simulations and experiments for ranolazine and terfenadine agreed for $\mathrm{dV} / \mathrm{dt}_{\mathrm{MAX}}$ at every pacing frequency and qualitatively for $\mathrm{APD}_{90}$ at slow pacing. At higher frequencies human simulations showed $\mathrm{AP}$ prolongation larger than in rabbit experiments. Simulated drug-induced $\mathrm{APD}_{90}$ and $\mathrm{APD}_{50}$ prolongations for dofetilide (Figure $7 \mathrm{C}$ ) and sotalol were significantly smaller than those observed experimentally in rabbit, at all tested concentrations and pacing frequencies, due to well-known species differences in hERG block sensitivity as discussed in Section 3.3. 


\section{Biomarkers consistency between human simulations and rabbit experiments}

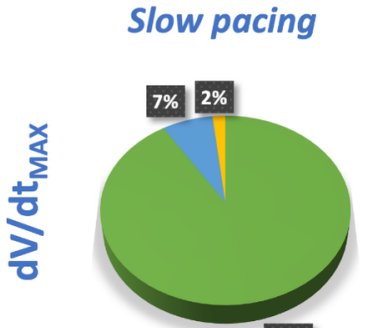

$91 \%$
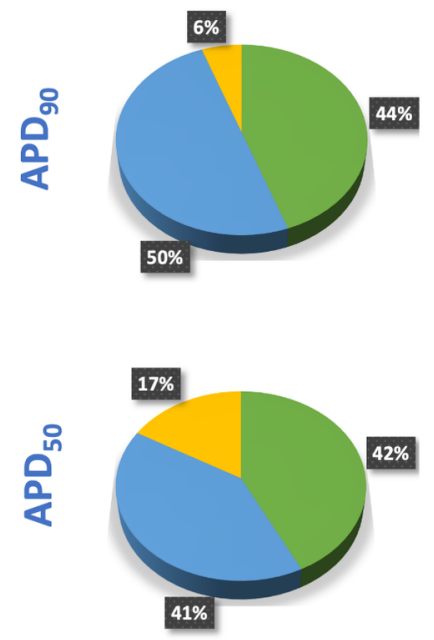

Normal pacing
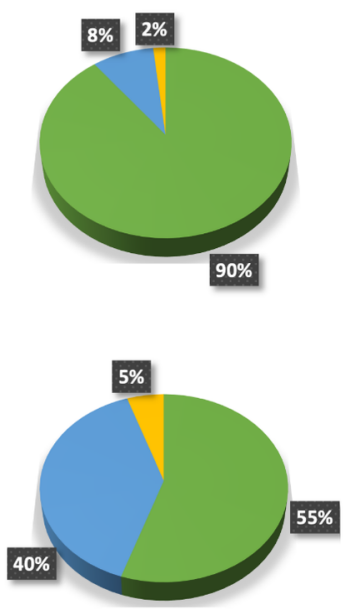

$24 \%$

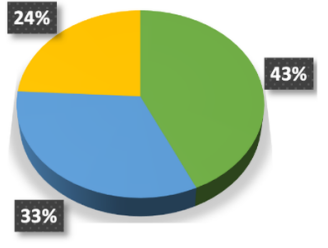

$17 \%$

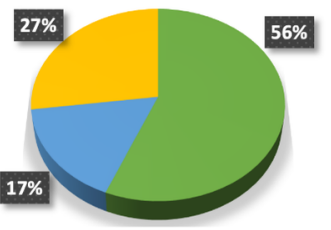

Fast pacing
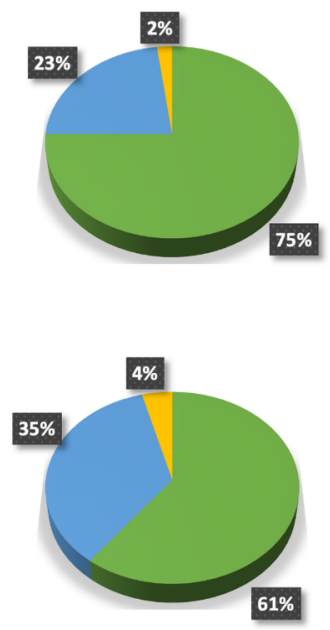

$61 \%$

Strong Agreement Qualitative agreement Disagreement

Figure 6. Summary of the comparison between experiments and simulations at different pacing frequencies, for $\mathrm{dV} / \mathrm{dt}_{\mathrm{MAX}}$ (top), $\mathrm{APD}_{90}$ (middle), and $\mathrm{APD}_{50}$ (bottom). Pie charts show the percentage of tested compounds at different concentrations in strong agreement (green), qualitative agreement (blue), or disagreement (yellow). Categories defined as in Section 2.3). 
A Disopyramide
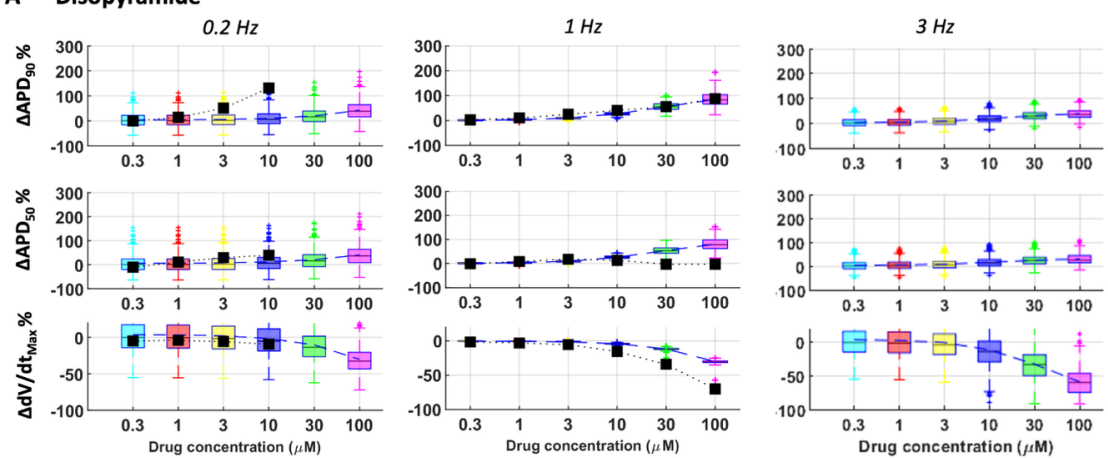

B Bepridil
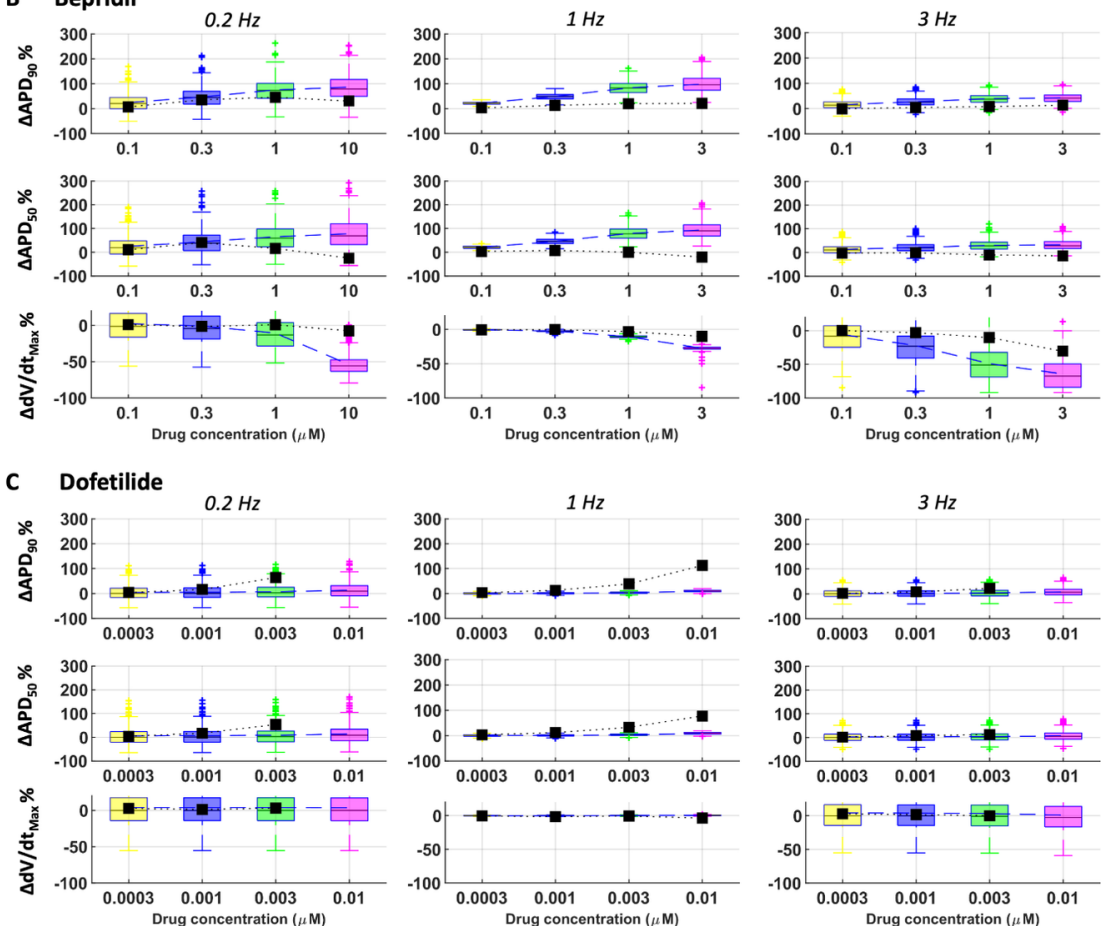

Figure 7. Comparison between simulated and experimental dose-response curves for $\mathrm{APD}_{90}, \mathrm{APD}_{50}$ and $\mathrm{dV} / \mathrm{dt}_{\text {MAX }}$, for three representative compounds, at all pacing frequencies: A) disopyramide; B) bepridil; C) dofetilide. Boxplots: human simulations at different concentrations (one colour per concentration): on each box, the central mark is the median of the population, box limits are the 25 and 75 th percentiles, and whiskers extend to the most extreme data points not considered outliers, plotted individually as separate crosses. Black squares: in vitro rabbit data.

\section{DISCUSSION AND CONCLUSION}

In this study, we showcase the large impact that human in silicodrug trials can have in the context of predictions of drug-induced proarrhythmic risk based on ion channel information, by providing new evidence 
obtained through simulations in human cardiac Purkinje cells. We present results for a selection of 14 reference compounds (at multiple concentrations and pacing rates), using in silico human Purkinje models with a variety of ionic profiles $(\mathrm{n}=530)$, their comparison to in vitro rabbit Purkinje fibres experiments $(\mathrm{n}=6)$, and their ability to predicting clinical risk, based on various biomarkers, including EADs occurrence and $\mathrm{APD}_{90}$.

The main findings of this study are:

1. In silico predictions using human Purkinje models based on EAD occurrence at slow pacing showed $100 \%$ accuracy in classifying risky from safe drugs, while in vitro rabbit experiments yielded $79 \%$ accuracy. This was also superior to predictions based on AP prolongation, which yielded accuracy of $86 \%$ for both in silicoand in vitro .

2. In silico drug trials using human cardiac Purkinje electrophysiology models and in vitro rabbit Purkinje recordings showed a high degree of consistency for all tested compounds, across biomarkers, concentrations and pacing frequencies. This supports the credibility of human-based in silicomodelling and simulations for the replacement of animal experiments in this context of use.

3. Some compounds, e.g., clarithromycin, dofetilide, sotalol and terfenadine, displayed a larger AP prolongation in vitro rabbit compared to in silico human recordings. This is in agreement with well-known differences between rabbits and humans in the response to hERG block.

The high translatability of human-based in silico drug trials to clinical outcome - as demonstrated here for electrophysiology -highlights their potential for high regulatory impact in drug discovery (Musuamba et al. 2021). Human-based computational simulations can accurately predict clinical drug-induced arrhythmia (Passini et al. 2017, 2019; Lancaster and Sobie 2016; Llopis-Lorente et al. 2020; Z. Li, Ridder, et al. 2019). This is particularly relevant for compounds with positive hERG assays that may not induce arrhythmia due to their concomitant effect on $\mathrm{I}_{\mathrm{Na}}$ and $\mathrm{I}_{\mathrm{CaL}}$.

We previously demonstrated how human in silico trials using ventricular cardiomyocytes reach higher prediction accuracy than animal models for drug-induced pro-arrhythmia (Passini et al. 2017), and also their consistency with recordings from isolated rabbit wedge and calcium transients from human induced pluripotent stem cell-derived cardiomyocytes (hiPSC-CMs). Here we compare proarrhythmic risk predictions and drug-induced electrophysiological changes in humanin silico cardiac Purkinje cells against and rabbit in vitro Purkinje fibres, which is a well-established model for preclinical safety assessments in pharmaceutical industries.

For preclinical risk assessment, we first considered a metric based on drug induced repolarisation abnormalities occurrences at slow pacing, similar to (Passini et al. 2017; Varshneya, Mei, and Sobie 2021; Sager et al. 2014). We reached an accuracy of $100 \%$ using human in silico drug trials, compared to only $86 \%$ using in vitrorecordings, which failed to identify two compounds with known TdP risk (bepridil and terfenadine) and one with conditional TdP risk (ranolazine). Our findings are in agreement with previous experimental studies, showing that these three compounds often did not show EADs when tested in rabbit preparations. Bepridil up to $10 \mu \mathrm{M}$ did not induce EADs in rabbit hearts (Hondeghem et al. 2003; Anno et al. 1984), while it did on hiPSC-CMs at the same concentration (Yu et al. 2019). Similarly, terfenadine did not induced EADs in rabbit wedge preparations (Vos 2008; Liu et al. 2006), but it did in hiPSC-CMs (Nozaki et al. 2014). Ranolazine, which is associated with TdP only under certain conditions, e.g. hypokalaemia, bradycardia, etc. (Woosley and Romer 1999), has shown anti-torsadogenic effects in rabbit hearts at $10 \mu \mathrm{M}$ (Sossalla et al. 2014; Frommeyer et al. 2012), but at $100 \mu \mathrm{M}$ induced EADs on hiPSC-CMs (Blinova et al. 2018; Yu et al. 2019).

The differences in drug-induced EADs occurrence between in silicohuman and in vitro rabbit results are likely to be due to the clear advantage of in silico simulations performed in 530 virtual myocytes, rather than a limited number of experiments $(\mathrm{n}=6)$. In addition, the in silico population of models incorporates a large variability in ionic profiles (over- and under-expression of ionic currents), and we previously demonstrated that models with low repolarisation reserve are more likely to develop EADs following ion channel blocks, 
both in ventricular and cardiac Purkinje models (Trovato et al. 2020; Passini et al. 2017). Therefore, it is much more likely to observe drug-induced EADs in human in silico drug trials. This is a clear advantage when trying to predict risk.

We also evaluated how in silico and in vitro predictions based on drug-induced AP prolongation compare against clinical risk, since APD is still one of the most common biomarkers considered in preclinical safety, despite several studies showed that it is not always associated with TdP risk, especially for multichannel blockers (Champeroux et al. 2005; Redfern et al. 2003). In our study, predictions based on $\mathrm{APD}_{90}$ at $1 \mathrm{~Hz}$ reached the same accuracy (86\%) in silico and in vitro, and results were highly consistent (>90\%) across all drugs and all concentrations.

Quantitative comparison of drug-induced changes for all biomarkers $\left(\mathrm{dV} / \mathrm{dt}_{\mathrm{MAX}}, \mathrm{APD}_{90}\right.$, and $\left.\mathrm{APD}_{50}\right)$ and pacing frequencies between human simulations and rabbit preparations also showed large consistency. This is a very exciting result, confirming the importance of developing in silico models using experimental data at different frequencies, as we did for the human cardiac Purkinje models used in this study (Trovato et al. 2020).

Some compounds, e.g., clarithromycin, dofetilide, sotalol and terfenadine, displayed a larger AP prolongation in vitro compared to in silico, due to well-known differences between rabbits and humans in responding to hERG block. Previous studies have reported smaller clarithromycin-induced AP prolongation in humans compared to rabbit (Gluais et al. 2003), and no QT prolongation at therapeutic doses (Démolis et al. 2003). Also, several studies have reported in rabbit the largest dofetilide-induced AP prolongation, compared to other species e.g. humans, dog, guinea pig, swine, goat, sheep (Lu et al. 2002, 2001; Terrar et al. 2007; Trovato et al. 2020). Previous studies also showed larger sotalol-induced AP prolongation in rabbit compared to other species (Gintant et al. 2001), and AP prolongation between $28 \%$ and $37 \%$ following superinfusion of sotalol $30 \mu \mathrm{M}$ in human cardiomyocytes (Tveito et al. 2020), closer to what observed in our simulations (16\%) than in rabbit experiments (96\%).

To our knowledge, this is the first study that systematically evaluates and compares homogenous experimental data capturing drug-induced effect on cardiac Purkinje fibres against in silico results. To minimise noise and variability in the experimental electrophysiological recordings, we considered a consistent dataset, with experiments performed in one laboratory and under identical conditions.

In summary, the credibility goals that we satisfied in this study, as defined in (Musuamba et al. 2021), are: i) to show higher accuracy ofin silico trials compared to a current-in-use experimental counterpart; ii) to demonstrate high grade of consistency between simulations and experiments. Our results showed not only high degree of consistency between in vitro and in silico preparations (Figure 6), but also that humanbased computer simulations can achieve better results than rabbit experiments for risk predictions (Figure 4A) since they are built, calibrated, and validated using human data, thus facilitating translation towards clinical scenarios.

In addition, there are many more advantages in using in silicomodel compared to perform in vitro animal experiments. These include: i) reduction of the use of animals in research; ii) reduction of the time required for drug safety assessment, thus allowing pharma companies to process more compounds in a shorter amount of time, and accelerate the drug development process; iii) economical advantage, i.e.in silico trials can be performed in a standard computer; iv) overcome limitations in the tested concentration ranges, due to drug solubility problems; v) overcome limitations in the number of conditions explored for each drug (concentrations, pacing frequencies, etc.) and in the number of samples.

To conclude, in silico drug trials in human cardiac Purkinje cells have shown to be consistent with in vitro recordings from rabbit Purkinje fibres, and more accurate for predictions of drug-induced proarrhythmic risk. This supports the opportunity for replacement of animal experiments with human-based in silicosimulations, in the regulatory context.

\section{REFERENCES}


Anno, Takafumi, Tatsuji Furuta, Masanori Itoh, Itsuo Kodama, Junji Toyama, and Kazuo Yamada. 1984. "Electromechanical Effects of Bepridil on Rabbit Isolated Hearts." British Journal of Pharmacology 81 (1): 41-47. https://doi.org/10.1111/j.1476-5381.1984.tb10741.x.

Blinova, Ksenia, Qianyu Dang, Daniel Millard, Norman Stockbridge, David G Strauss, Gary Gintant, Ksenia Blinova, et al. 2018. "International Multisite Study of Human-Induced Pluripotent Stem Cell-Derived Cardiomyocytes for Drug Proarrhythmic Potential Assessment Resource International Multisite Study of Human-Induced Pluripotent Stem Cell-Derived Cardiomyocytes for Drug Proarrhyt." Cell Reports 24 (13): 3582-92. https://doi.org/10.1016/j.celrep.2018.08.079.

Britton, Oliver J., Alfonso Bueno-Orovio, Karel Van Ammel, Hua Rong Lu, Rob Towart, David J. Gallacher, and Blanca Rodriguez. 2013. "Experimentally Calibrated Population of Models Predicts and Explains Intersubject Variability in Cardiac Cellular Electrophysiology." Proceedings of the National Academy of Sciences 110 (23): E2098-2105. https://doi.org/10.1073/pnas.1304382110.

Champeroux, Pascal, Karen Viaud, Abdel Ilah El Amrani, John Sinclair Lawrence Fowler, Eric Martel, Jean-Yves Le Guennec, and Serge Richard. 2005. "Prediction of the Risk of Torsade de Pointes Using the Model of Isolated Canine Purkinje Fibres." British Journal of Pharmacology 144 (3): 376-85. https://doi.org/10.1038/sj.bjp.0706070.

Cohen, C. J., B. P. Bean, and R. W. Tsien. 1984. "Maximal Upstroke Velocity as an Index of Available Sodium Conductance. Comparison of Maximal Upstroke Velocity and Voltage Clamp Measurements of Sodium Current in Rabbit Purkinje Fibers." Circulation Research 54 (6): 636-51. https://doi.org/10.1161/01.RES.54.6.636.

Crumb, William J., Jose Vicente, Lars Johannesen, and David G. Strauss. 2016. "An Evaluation of 30 Clinical Drugs against the Comprehensive in Vitro Proarrhythmia Assay (CiPA) Proposed Ion Channel Panel." Journal of Pharmacological and Toxicological Methods 81 (2): 251-62. https://doi.org/10.1016/j.vascn.2016.03.009.

Démolis, Jean Louis, Françoise Vacheron, Stéphane Cardus, and Christian Funck-Brentano. 2003. "Effect of Single and Repeated Oral Doses of Telithromycin on Cardiac QT Interval in Healthy Subjects." Clinical Pharmacology and Therapeutics 73 (3): 242-52. https://doi.org/10.1067/mcp.2003.4.

EMEA. 2006. "ICH Topic S 7 B : The Nonclinical Evaluation of the Potential for Delayed Ventricular Repolarization (QT Interval Prolongation) by Human Pharmaceuticals." European Medicines Agency, no. December 2004: 1-9.

Frommeyer, Gerrit, Dennis Kaiser, Timo Uphaus, Sven Kaese, Nani Osada, Sridharan Rajamani, Luiz Belardinelli, Gunter Breithardt, Lars Eckardt, and Peter Milberg. 2012. "Effect of Ranolazine on Ventricular Repolarization in Class III Antiarrhythmic Drug-Treated Rabbits." Heart Rhythm 9 (12): 2051-58. https://doi.org/10.1016/j.hrthm.2012.08.029.

Gintant, G a, J T Limberis, J S McDermott, C D Wegner, and B F Cox. 2001. "The Canine Purkinje Fiber: An in Vitro Model System for Acquired Long QT Syndrome and Drug-Induced Arrhythmogenesis." Journal of Cardiovascular Pharmacology 37 (5): 607-18. https://doi.org/10.1097/00005344-200105000-00012.

Gintant, Gary, Philip T. Sager, and Norman Stockbridge. 2016. "Evolution of Strategies to Improve Preclinical Cardiac Safety Testing." Nature Reviews Drug Discovery 15 (7): 457-71. https://doi.org/10.1038/nrd.2015.34.

Gluais, Pascale, Michele Bastide, Daniel Grandmougin, Georges Fayad, and Monique Adamantidis. 2003. "Clarithromycin Reduces Isus and Ito Currents in Human Atrial Myocytes with Minor Repercussions on Action Potential Duration." Fundamental and Clinical Pharmacology 17 (6): 691-701. https://doi.org/10.1046/j.1472-8206.2003.00186.x. 
Hondeghem, Luc M., Hua Rong Lu, Koen Van Rossem, and Fred De Clerck. 2003. "Detection of Proarrhythmia in the Female Rabbit Heart: Blinded Validation." Journal of Cardiovascular Electrophysiology 14 (3): 287-94. https://doi.org/10.1046/j.1540-8167.2003.02466.x.

Kramer, James, Carlos A. Obejero-Paz, Glenn Myatt, Yuri A Kuryshev, Andrew Bruening-Wright, Joseph S Verducci, and Arthur M Brown. 2013. "MICE Models: Superior to the HERG Model in Predicting Torsade de Pointes." Scientific Reports 3 (1): 2100. https://doi.org/10.1038/srep02100.

Lancaster, M. Cummins, and E. A. Sobie. 2016. "Improved Prediction of Drug-Induced Torsades de Pointes Through Simulations of Dynamics and Machine Learning Algorithms." Clinical Pharmacology and Therapeutics 100 (4): 371-79. https://doi.org/10.1002/cpt.367.

Lester, Robert M., and Joy Olbertz. 2016. "Early Drug Development: Assessment of Proarrhythmic Risk and Cardiovascular Safety: The Age of Repolarization Cardiac Toxicity." Expert Review of Clinical Pharmacology 9 (12): 1611-18. https://doi.org/10.1080/17512433.2016.1245142.

Li, Wei, Ying Yu, Jian-Wen Hou, Zhi-wen Zhou, Kai Guo, Peng-Pai Zhang, Zhi-Quan Wang, et al. 2016. "Larger Rate Dependence of Late Sodium Current in Cardiac Purkinje Cells: A Potential Link to Arrhythmogenesis." Heart Rhythm 14 (3): 1-10. http://linkinghub.elsevier.com/retrieve/pii/S1547527116311559.

Li, Zhihua, Gary R Mirams, Takashi Yoshinaga, Bradley J Ridder, Xiaomei Han, Janell E Chen, Norman L Stockbridge, et al. 2019. "General Principles for the Validation of Proarrhythmia Risk Prediction Models: An Extension of the CiPA In Silico Strategy." Clinical Pharmacology $\mathscr{E}$ Therapeutics 0 (0): cpt.1647. https://doi.org/10.1002/cpt.1647.

Li, Zhihua, Bradley J. Ridder, Xiaomei Han, Wendy W. Wu, Jiansong Sheng, Phu N. Tran, Min Wu, et al. 2019. "Assessment of an In Silico Mechanistic Model for Proarrhythmia Risk Prediction Under the CiPA Initiative." Clinical Pharmacology and Therapeutics 105 (2): 466-75. https://doi.org/10.1002/cpt.1184.

Liu, Tengxian, Barry S. Brown, Ying Wu, Charles Antzelevitch, Peter R. Kowey, and Gan Xin Yan. 2006. "Blinded Validation of the Isolated Arterially Perfused Rabbit Ventricular Wedge in Preclinical Assessment of Drug-Induced Proarrhythmias." Heart Rhythm 3 (8): 948-56. https://doi.org/10.1016/j.hrthm.2006.04.021.

Llopis-Lorente, Jordi, Julio Gomis-Tena, Jordi Cano, Lucia Romero, Javier Saiz, and Beatriz Trenor. 2020. "In Silico Classifiers for the Assessment of Drug Proarrhythmicity." Journal of Chemical Information and Modeling 60 (10): 5172-87. https://doi.org/10.1021/acs.jcim.0c00201.

Lu, Hua Rong, Roger Marie, A N N Saels, and Fred D E Clerck. 2001. "Species Plays an Important Role in Drug-Induced Prolongation of Action Potential Duration and Early Afterdepolarizations in Isolated Purkinje Fibers" 12 (1): 93-102.

Lu, Hua Rong, Eddy Vlaminckx, Karel Van Ammel, and Fred De Clerck. 2002. "Drug-Induced Long QT in Isolated Rabbit Purkinje Fibers: Importance of Action Potential Duration, Triangulation and Early Afterdepolarizations." European Journal of Pharmacology 452 (2): 183-92. https://doi.org/10.1016/S00142999(02)02246-X.

McKay, M. D., R J Beckman, and W J Conover. 1979. "A Comparison of Three Methods for Selecting Values of Input Variables in the Analysis of Output from a Computer Code." Technometrics 21 (2): 239. https://doi.org/10.2307/1268522.

Musuamba, Flora T, Ine Skottheim Rusten, Raphaelle Lesage, Giulia Russo, Roberta Bursi, Luca Emili, Gaby Wangorsch, et al. 2021. "Scientific and Regulatory Evaluation of Mechanistic in Silico Drug and Disease Models in Drug Development: Building Model Credibility." CPT: Pharmacometrics \&5 Systems Pharmacology 10 (8): 804-25. https://doi.org/10.1002/psp4.12669.

Muszkiewicz, Anna, Oliver J Britton, Philip M. Gemmell, Elisa Passini, Carlos Sanchez, Xin Zhou, Annamaria Carusi, et al. 2015. "Variability in Cardiac Electrophysiology: Using Experimentally-Calibrated 
Populations of Models to Move beyond the Single Virtual Physiological Human Paradigm." Progress in Biophysics and Molecular Biology, 1-13. https://doi.org/10.1016/j.pbiomolbio.2015.12.002.

Nagy, N., T. Szel, N. Jost, A. Toth, J. Gy Papp, and A. Varro. 2015. "Novel Experimental Results in Human Cardiac Electrophysiology: Measurement of the Purkinje Fibre Action Potential from the Undiseased Human Heart." Canadian Journal of Physiology and Pharmacology810 (May): 1-8. https://doi.org/10.1139/cjpp2014-0532.

Norman, Gail A. Van. 2019. "Limitations of Animal Studies for Predicting Toxicity in Clinical Trials: Is It Time to Rethink Our Current Approach?" JACC: Basic to Translational Science 4 (7): 845-54. https://doi.org/10.1016/j.jacbts.2019.10.008.

Nozaki, Yumiko, Yayoi Honda, Shinji Tsujimoto, Hitoshi Watanabe, Takeshi Kunimatsu, and Hitoshi Funabashi. 2014. "Availability of Human Induced Pluripotent Stem Cell-Derived Cardiomyocytes in Assessment of Drug Potential for QT Prolongation." Toxicology and Applied Pharmacology 278 (1): 72-77. https://doi.org/10.1016/j.taap.2014.04.007.

Passini, Elisa, Oliver J. Britton, Hua Rong Lu, Jutta Rohrbacher, An N. Hermans, David J. Gallacher, Robert J. H. Greig, Alfonso Bueno-Orovio, and Blanca Rodriguez. 2017. "Human In Silico Drug Trials Demonstrate Higher Accuracy than Animal Models in Predicting Clinical Pro-Arrhythmic Cardiotoxicity." Frontiers in Physiology 8 (September): 1-15. https://doi.org/10.3389/fphys.2017.00668.

Passini, Elisa, Cristian Trovato, Pierre Morissette, Frederick Sannajust, Alfonso Bueno-Orovio, and Blanca Rodriguez. 2019. "Drug-induced Shortening of the Electromechanical Window Is an Effective Biomarker for in Silico Prediction of Clinical Risk of Arrhythmias." British Journal of Pharmacology 176 (19): 3819-33. https://doi.org/10.1111/bph.14786.

Passini, Elisa, Xin Zhou, Cristian Trovato, Oliver J. Britton, Alfonso Bueno-Orovio, and Blanca Rodriguez. 2021. "The Virtual Assay Software for Human in Silico Drug Trials to Augment Drug Cardiac Testing." Journal of Computational Science 52 (May 2020): 101202. https://doi.org/10.1016/j.jocs.2020.101202.

Redfern, W, L Carlsson, A Davis, W Lynch, I Mackenzie, S Palethorpe, P Siegl, I Strang, A Sullivan, and R Wallis. 2003. "Relationships between Preclinical Cardiac Electrophysiology, Clinical QT Interval Prolongation and Torsade de Pointes for a Broad Range of Drugs: Evidence for a Provisional Safety Margin in Drug Development." Cardiovascular Research 58 (1): 32-45. https://doi.org/10.1016/S0008-6363(02)008465 .

Roche, Michel, C??line Celine Renauleaud, Veronique V??ronique Ballet, Michel Doubovetzky, and Jean Michel Guillon. 2010. "The Isolated Rabbit Heart and Purkinje Fibers as Models for Identifying Proarrhythmic Liability." Journal of Pharmacological and Toxicological Methods61 (3): 238-50. https://doi.org/10.1016/j.vascn.2010.01.011.

Sager, Philip T., Gary Gintant, J. Rick Turner, Syril Pettit, and Norman Stockbridge. 2014. "Rechanneling the Cardiac Proarrhythmia Safety Paradigm: A Meeting Report from the Cardiac Safety Research Consortium." American Heart Journal 167 (3): 292-300. https://doi.org/10.1016/j.ahj.2013.11.004.

Sossalla, Samuel, Nora Wallisch, Karl Toischer, Christian Sohns, Dirk Vollmann, Joachim Seegers, Lars Luthje, Lars S. Maier, and Markus Zabel. 2014. "Effects of Ranolazine on Torsades de Pointes Tachycardias in a Healthy Isolated Rabbit Heart Model." Cardiovascular Therapeutics 32 (4): 170-77. https://doi.org/10.1111/1755-5922.12078.

Terrar, Derek A., C. M. Wilson, S. G. Graham, S. M. Bryant, and B. M. Heath. 2007. "Comparison of Guinea-Pig Ventricular Myocytes and Dog Purkinje Fibres for in Vitro Assessment of DrugInduced Delayed Repolarization." Journal of Pharmacological and Toxicological Methods 56 (2): 171-85. https://doi.org/10.1016/j.vascn.2007.04.005.

Trovato, C., C. Dusserre, S. Billiald-Desquand, E. Passini, and B. Rodriguez. 2020. "In Silico Trials for 
Drug Safety and Efficacy Assessment Using a Novel Human Purkinje Fibre Model." Computing in Cardiology 2020-Septe: 10-13. https://doi.org/10.22489/CinC.2020.361.

Trovato, Cristian, Elisa Passini, Norbert Nagy, Andras Varro, Najah Abi-Gerges, Stefano Severi, and Blanca Rodriguez. 2020. "Human Purkinje in Silico Model Enables Mechanistic Investigations into Automaticity and Pro-Arrhythmic Abnormalities." Journal of Molecular and Cellular Cardiology 142 (April): 24-38. https://doi.org/10.1016/j.yjmcc.2020.04.001.

Tveito, Aslak, Karoline Horgmo Jaeger, Mary M. Maleckar, Wayne R. Giles, and Samuel Wall. 2020. "Computational Translation of Drug Effects from Animal Experiments to Human Ventricular Myocytes." Scientific Reports 10 (1): 1-11. https://doi.org/10.1038/s41598-020-66910-0.

Varshneya, Meera, Xueyan Mei, and Eric A. Sobie. 2021. "Prediction of Arrhythmia Susceptibility through Mathematical Modeling and Machine Learning." Proceedings of the National Academy of Sciences of the United States of America 118 (37). https://doi.org/10.1073/pnas.2104019118.

Vos, M. A. 2008. "Literature-Based Evaluation of Four ' Hard Endpoint' Models for Assessing Drug-Induced Torsades de Pointes Liability." British Journal of Pharmacology 7 (July): 1523-27. https://doi.org/10.1038/bjp.2008.277.

Woosley, RL, and KA Romer. 1999. "Www.Crediblemeds.Org, QTdrugs List [Accessed 2021-09-01]." AZCERT, Inc. 1822 Innovation Park Dr., Oro Valley, AZ 85755. 1999.

Yu, Yankun, Mengrong Zhang, Ren Chen, Feng Liu, Pengfei Zhou, Lei Bu, Ying Xu, and Lei Zheng. 2019. "Action Potential Response of Human Induced-Pluripotent Stem Cell Derived Cardiomyocytes to the 28 CiPA Compounds: A Non-Core Site Data Report of the CiPA Study." Journal of Pharmacological and Toxicological Methods 98 (October 2018): 106577. https://doi.org/10.1016/j.vascn.2019.04.003.

Zhou, Xin, Yusheng Qu, Elisa Passini, Alfonso Bueno-Orovio, Yang Liu, Hugo M. Vargas, and Blanca Rodriguez. 2020. "Blinded in Silico Drug Trial Reveals the Minimum Set of Ion Channels for Torsades de Pointes Risk Assessment." Frontiers in Pharmacology 10 (January): 1-17. https://doi.org/10.3389/fphar.2019.01643. 